\title{
Meta
}

Journal des traducteurs

Translators' Journal

\section{Bosseaux, C. (2007) : How Does It Feel ? Point of View in Translation, Amsterdam/New York, Rodopi, coll. “Approaches to Translation Studies”, volume 29, 247 p.}

\section{Serge Marcoux}

Volume 54, numéro 1, janvier 2009

URI : https://id.erudit.org/iderudit/029800ar

DOI : https://doi.org/10.7202/029800ar

Aller au sommaire du numéro

Éditeur(s)

Les Presses de l'Université de Montréal

ISSN

0026-0452 (imprimé)

1492-1421 (numérique)

Découvrir la revue

Citer ce compte rendu

Marcoux, S. (2009). Compte rendu de [Bosseaux, C. (2007) : How Does It Feel ? Point of View in Translation, Amsterdam/New York, Rodopi, coll. "Approaches to Translation Studies”, volume 29, 247 p.] Meta, 54(1), 164-170.

https://doi.org/10.7202/029800ar d'utilisation que vous pouvez consulter en ligne. 
Bosseaux, C. (2007): How Does It Feel? Point of View in Translation, Amsterdam/ New York, Rodopi, coll. “Approaches to Translation Studies”, volume 29, 247 p.

Depuis 2004, Charlotte Bosseaux enseigne la traduction à l'Université d'Édimbourg. Détentrice de plusieurs diplômes d'universités françaises et britanniques et auteure de plusieurs études sur Virginia Woolf, elle s'intéresse notamment à la théorie de la traduction, à l'utilisation des corpus en traductologie et à la narratologie.

Dans cet ouvrage, où se rencontrent traductologie et narratologie, elle tente d'analyser la relation existant entre les personnages d'un roman et l'univers fictif dans lequel ils se meuvent, ce que Paul Simpson appelait en anglais le feel d'un récit. Nous apprenons à connaître ces personnages soit par la voix d'un tiers, le narrateur omniscient, soit par les dialogues ou monologues qui les mettent directement en scène, nous offrant leur point de vue ou perspective (le point of view auquel réfère le titre) à la première ou à la troisième personne. Si la traduction entraîne une modification du caractère d'un personnage, son univers fictif s'en trouvera-t-il remanié et, le cas échéant, à quel point? C'est la question à laquelle l'auteure veut répondre en comparant, sur la base de corpus et de concordanciers, deux romans de Virginia Woolf, To the Lighthouse (1927) et The Waves (1931), et leurs traductions françaises.

La première partie de l'ouvrage (chapitres 1, 2 et 3) est donc consacrée à l'élaboration d'un modèle théorique permettant de retrouver sur le plan du lexique et du style les traces que peut laisser un traducteur dans un texte. Le chapitre 4 nous présente Virginia Woolf et les deux romans auxquels sera appliqué ce modèle théorique. Enfin, les chapitres 5 et 6 exposent les résultats de la recherche, le premier en se concentrant surtout sur l'utilisation du discours indirect libre dans To the Lighthouse; le deuxième sur les particularités lexicales et stylistiques des traducteurs dans The Waves.

Depuis quelques années, les spécialistes de la narratologie ont concentré leurs recherches sur la question: "Qui parle à qui ? ». Curieusement toutefois, ils ont traité sur le même pied œuvres originales et traductions, négligeant le fait que les œuvres traduites s'adressent à un public linguistiquement et culturellement différent de celui pour lequel l'auteur a écrit, situation dont le traducteur se doit de tenir compte. De telle sorte que, pour reprendre la terminologie des formalistes russes et des structuralistes français, si l'histoire demeure essentiellement la même, le discours, lui, peut s'en trouver substantiellement modifié avec les conséquences qui en découleront sur l'univers fictif du récit.

Au dix-neuvième siècle, les auteurs britanniques, Dickens en particulier, ont eu tendance à raconter l'histoire par la voix d'un narrateur omniprésent et omniscient. Les auteurs contemporains (pensons ici à Ian McEwan et à son Saturday) tentent plutôt de raconter leur histoire par le truchement des personnages qui nous dévoilent eux-mêmes leur caractère, leurs états d'âme et leurs pensées, ce qui a donné naissance à la technique du discours indirect libre, un discours indirect empruntant la forme du discours direct. Beaucoup plus fréquente en anglais qu'en français, cette technique peut difficilement se traduire littéralement et peut entraîner des glissements lorsque la voix du narrateur se superpose à celle d'un personnage.

Il peut arriver que la présence du traducteur se révèle ouvertement soit par des notes de bas de page ou par des étoffements lorsque les contextes culturels exigent des explications. Le plus souvent toutefois, c'est la structure interne d'une langue qui exigera de lui des modifications par rapport au texte original. Enfin, la présence du traducteur peut se cacher derrière celle du narrateur et son identification ne sera perçue qu'à travers le «style» propre du traducteur, sorte d'empreinte digitale composée de stratégies de traduction et de formes d'expression auxquelles celui-ci aura fréquemment recours lorsque confronté à un problème particulier.

Ayant ainsi défini dans un premier chapitre ce qu'est le point de vue ou perspective et sa relation avec l'univers fictif d'un récit, l'auteure énumère et définit au chapitre 2 les 
différentes perspectives que l'on peut trouver dans un texte de fiction: spatiale, temporelle, psychologique et idéologique.

La perspective spatiale est celle adoptée par le narrateur de l'histoire; la perspective temporelle, celle dans laquelle l'auteur circonscrit la trame de l'action. Prises conjointement, elles constituent la perspective spatio-temporelle ou deixis du texte. La perspective psychologique découle de la façon dont le narrateur évalue les faits qu'il raconte. Enfin, la perspective idéologique réfère au système de valeurs et de croyances inhérent à l'ensemble du texte.

L'impression d'ensemble qui se dégage de l'univers fictif du récit découle de la perspective adoptée autant pour ce qui a trait au texte original qu'en ce qui a trait à la traduction. L'auteure étudie donc dans ce chapitre comment comparer texte original et traduction pour déterminer (1) si la traduction reste fidèle à l'original ou si on y trouve des glissements, (2) si l'univers fictif du texte original et celui de la traduction sont identiques, (3) comment cet univers sera perçu par les lecteurs du texte original et ceux de la traduction.

La perspective spatio-temporelle ou deixis fournira une première base de comparaison. Le langage est plus qu'une suite de mots arrangés selon un certain ordre grammatical. Il permet d'identifier différents êtres ou choses et de les mettre en rapport avec une situation donnée. Les pronoms personnels (moi, tu, elle, etc.), les adjectifs déterminatifs, permettent ainsi de relier les aspects subjectifs associés aux acteurs et les aspects objectifs associés aux situations auxquelles ils sont confrontés. Dans un tel système, le «je» devient ainsi le point central qui transforme le langage en discours puisque toutes les situations s'ordonneront en fonction de cet élément. Les adverbes, comme ici ou là, maintenant ou jamais, préciseront comment une situation s'articule autour de lui dans l'espace, alors que les verbes joueront le même rôle dans le temps. Ce sont ces éléments qui situent l'action au sein du monde dans lequel nous vivons et qui permettent de transposer l'action dans le monde fictif où se situe tout texte narratif.

Dans une traduction, deux difficultés méritent considération. La première, de nature linguistique, tient à la structure même des deux langues en question: le futur par exemple s'exprime de façon différente en français et en allemand. La deuxième, de nature psychologique, tient au fait que le traducteur doit deviner ce qu'a voulu dire l'auteur en utilisant ces termes subjectifs, tâche d'autant plus difficile que des centaines d'années ou de kilomètres peuvent le séparer de l'auteur.

Une deuxième base de comparaison est la modalité, c'est-à-dire la façon dont les relations se créent, se poursuivent ou se modifient entre les membres d'une société. Elle inclut les jugements sur la réalité des faits, les obligations des partenaires, les valeurs des personnages et se traduit dans un texte par des nuances dans le choix des verbes (désirer, pouvoir, devoir) ou le choix des adverbes (assurément, certainement, probablement, peut-être, jamais).

De la même manière, la troisième base de comparaison ou transitivité réfère à la façon dont le narrateur ou les personnages encodent leur perception de la réalité dans le langage. On y distinguera donc (1) l'action ou l'état exprimés par le verbe de la phrase, (2) les participants à cette action ou l'état représentés par le sujet de la phrase ainsi que les attributs ou compléments directs et indirects et (3) les circonstances entourant l'action ou l'état telles que présentées par les compléments circonstanciels et les adverbes gravitant autour du verbe.

Quatrième et dernière base: le discours indirect libre, procédé stylistique sophistiqué permettant d'exprimer la vie intérieure des personnages d'un roman. En anglais, il consiste à faire appel au style indirect, mais en omettant les verbes et conjonctions qui y sont normalement associés.

Was she wrong in this, she asked herself, reviewing her conduct for the past week or two, and wondering if she had indeed put any pressure upon Minta, who was only twenty-four, to make up her mind. She was uneasy. Had she not laughed about it? Was she not forgetting again how strongly she influenced people? (Woolf 1927: 70) 
En français, certains auteurs comme Flaubert, Daudet, Zola et Maupassant utilisent le même procédé en passant dans une même phrase du passé simple à l'imparfait comme en témoigne l'exemple suivant:

En vain il parla de la sauvagerie du pays et de la difficulté pour une femme d'y voyager: elle ne craignait rien; elle aimait par-dessus tout à voyager à cheval; elle se faisait une fête de coucher au bivouac; elle menaçait d'aller en Asie Mineure. (dans Colomba de Prosper Mérimée, cité par Roy 1977)

Ayant ainsi défini la notion de perspective et les différents moyens permettant de l'identifier tant dans le texte original que dans une traduction, l'auteure consacre le troisième chapitre aux instruments méthodologiques élaborés pour mettre ces moyens en lumière.

La première partie du chapitre décrit diverses sortes de corpus et de concordanciers, ainsi que les instruments et logiciels mis au point pour les interroger. Particulièrement intéressants aux fins du présent ouvrage sont les corpus parallèles où, à un corps de textes dans une langue, se juxtapose la traduction dans une autre langue. Ceux-ci permettent notamment de constater les différences entre traductions ainsi que l'apparition de techniques particulières chez un traducteur. Les deux autres parties du chapitre sont consacrées aux modèles élaborés par Baker (2000) et Munday $(1998 ; 2002)$. Alors que Baker met surtout l'accent sur la recherche de traits distinctifs propres à un traducteur, Munday cherche plutôt à identifier les «glissements» introduits dans la traduction.

Mais peu importe la ou les méthodes, l'auteure souligne que l'utilisation de ces corpus ne doit pas viser à donner à la recherche une pseudo-objectivité scientifique. Toute recherche tend à prouver ou à infirmer une hypothèse basée sur un jugement humain; le processus d'interprétation demeure toujours subjectif et l'interprétation des données peut varier d'un chercheur à l'autre. Ils demeurent toutefois des outils extrêmement utiles en permettant de consulter une masse de données en un temps record et d'en extraire une quantité d'informations que seule limite l'habileté du chercheur à bien poser les questions et à traduire celles-ci en un langage accepté par l'ordinateur.

Le chapitre 4 constitue en quelque sorte un chapitre charnière. Après avoir décrit son modèle théorique, l'auteure nous présente Virginia Woolf, les traductions des deux romans cibles, To the Lighthouse et The Waves, ainsi que les critiques adressées à leurs traducteurs.

Virginia Woolf a, de façon consciente, cherché à renouveler la technique du roman. Elle a remplacé la notion d'identité, définie par les romanciers réalistes et édouardiens comme quelque chose d'extérieur et de définissable, par un concept beaucoup plus intérieur, ce qu'elle appelait «l'enveloppe à demi transparente» de la conscience (Woolf 1939). Dans tous ses romans, elle a voulu montrer à quel point la surface apparente des choses cachait la réalité de la vie, cette réalité qui n'est pas quelque chose d'objectif, mais plutôt une dynamique où personnages et environnement s'influencent mutuellement. Même les descriptions de paysages, morceaux de bravoure existant souvent par eux-mêmes chez les édouardiens, n'ont cours chez Virginia Woolf qu'en relation avec le caractère des personnages.

Pour y arriver, elle a utilisé une nouvelle technique narrative appelée stream of consciousness, ou courant de conscience, technique littéraire élaborée par William James en 1890 et qui cherche à décrire le point de vue d'un individu en donnant l'équivalent écrit du processus de pensée du personnage.

Publié en 1927, To the Lighthouse se situe dans une petite île des Hébrides où vit le couple Ramsay, leurs enfants et quelques invités. La Première Guerre mondiale viendra bouleverser ce tableau idyllique: Mme Ramsay décède, son fils est tué à la guerre et sa fille meurt en donnant naissance à un enfant. Dix ans plus tard, quelques survivants de la famille et leurs amis viennent revisiter ce qui fut un cadre de vie enchanteur. Le roman explore la conscience des acteurs du drame grâce à un ingénieux entrecroisé constitué des réactions 
des autres personnages, aux commentaires du narrateur et aux réflexions que font ces mêmes personnages les uns sur les autres.

Les trois traductions analysées par Charlotte Bosseaux ont été réalisées respectivement par Maurice Lanoire en 1929 sous le titre Promenade au phare, par Magali Merle, une spécialiste de Woolf, en 1993 sous le titre Voyage au phare et par Françoise Pellan, professeure à l'Université de Bourgogne et publié en 1996 sous le titre Vers le phare. La deuxième traduction fut rendue nécessaire lors de la publication des œuvres complètes de Virginia Woolf parce que celle de Lanoire avait été jugée maladroite et reflétant un mode d'esprit d'avantguerre disparu depuis. De la deuxième, celle de Merle, les éditeurs dirent qu'elle trahissait "une certaine transparence laissant deviner l'original sous les mots français (Reynier 1993)», ce qui constituait à leurs yeux un compliment. Pellan, quant à elle, avouait qu'elle avait tenté de suivre d'aussi près que possible la structure du style de Woolf et de son dynamisme interne.

The Waves retrace la vie de six personnages, Bernard, Jinny, Louis, Neville, Rhoda et Susan, de leur jeunesse à leur vieillesse. Le roman se veut, selon les mots de Woolf, une synthèse entre le roman, la poésie et le théâtre. Woolf y utilise fréquemment le monologue pour créer l'impression que les états d'âme du personnage sont communiqués directement au lecteur sans recours à un narrateur. Mais alors que dans le théâtre élisabéthain cette technique était le fait d'un personnage qui, à la dérobée, se retirait de l'action pour expliquer au spectateur la toile de fond d'un geste ayant déjà eu lieu, ici, le personnage demeure dans le feu de l'action et son monologue intérieur suggère une communication télépathique avec le lecteur.

Deux traductions en furent faites, la première en 1937 par Marguerite Yourcenar sous le titre Les Vagues et la deuxième, portant le même titre, en 1993 par Cécile Wajsbrot. Ces deux traductions sont l'antithèse l'une de l'autre. Autant celle de Yourcenar est celle d'une grande dame de la littérature qui «francise» la langue anglaise ${ }^{1}$, autant celle de Wajsbrot tente de rester fidèle au texte initial en utilisant force calques et en «transplantant» certaines formes syntaxiques, ce qui lui valut d'être critiquée par les uns pour avoir rendu le texte presque inintelligible (Forrester 1993) et louangée par les autres pour avoir permis au lecteur de lire de plain-pied le texte original en «transférant la langue étrangère dans la langue cible» (Cortanze 1993: 95-96). De telle sorte que derrière la traduction de Wajsbrot on n'entend que la voix de Woolf, alors que derrière celle de Yourcenar on n'entend que celle de Yourcenar.

Ces critiques étaient-elles justifiées? Comment évaluer si la perspective de Virginia Woolf a été respectée par les traducteurs ou jusqu'à quel point elle a été modifiée? Appliquant les critères de deixis, de modalité et de transitivité dans The Waves et du discours indirect libre dans To the Lighthouse, l'auteure explore les diverses hypothèses qu'elle tentera ensuite de démontrer dans les deux chapitres suivants en appliquant un modèle statistique aux deux corpus.

Le chapitre 5 est donc consacré à analyser les choix structuraux et syntaxiques retenus par les différents traducteurs de To the Lighthouse pour découvrir s'ils ont eu un impact sur l'homogénéisation de la structure narrative et sur l'univers fictif du récit. L'analyse statistique permet en même temps de vérifier à quel point le concordancier Multiconcord présente un avantage dans la localisation de certains mots clés.

Dans les chapitres précédents, divers indicateurs avaient été identifiés comme permettant de découvrir les passages en style indirect libre. Il s'agissait de certaines exclamations comme oh, yes et of course, de certaines interrogations comme but why, but how, de l'adverbe de temps now utilisé avec des verbes au passé ou au conditionnel et de certains adverbes dénotant un débat intérieur comme surely, certainly et perhaps.

Grâce au concordancier, l'auteure a ainsi pu identifier 162 indices de discours indirect libre dans les trois traductions proposées. Prometteurs au départ, ces indices se sont avérés 
limités à l'analyse, car les glissements identifiés étaient relativement mineurs. Toutefois, ils ont permis de constater qu'il règne dans la traduction de Pellan un certain degré d'incertitude sur l'identité du personnage qui parle, de telle sorte que la perspective de la traduction demeure très près du texte original où les voix des personnages et du narrateur se mélangent. Il en va de même de la traduction de Merle. Même si celle-ci est un peu plus uniforme, elle maintient l'incertitude sur l'identité de la personne qui parle. La traduction de Lanoire, pour sa part, donne une idée beaucoup plus claire de la distinction entre la voix des personnages et celle du narrateur, contribuant ainsi à homogénéiser le récit.

La deuxième partie de ce chapitre est consacrée à l'analyse de sept paragraphes contenant différents indices de discours indirect libre, paragraphes choisis parce que semblant particulièrement représentatifs des stratégies de Woolf, tout en demeurant difficiles d'identification en raison de la subtilité et des nuances de cet outil stylistique.

Ici également, la traduction de Lanoire se démarque des deux autres, le traducteur choisissant à l'occasion de transformer le discours indirect libre en discours direct ou indirect. Il en résulte que la traduction de Lanoire ne nous donne pas un accès aussi direct au monde intérieur des intervenants; leurs voix sont plus distinctes de celle du narrateur. Des trois, c'est la traduction de Pellan qui demeure la plus conforme à l'original. Ces quelques distorsions suffisent-elles à modifier la perspective communiquée par Woolf? L'auteure avoue qu'il y a matière à interprétation individuelle. Une chose est certaine toutefois: des trois traductions, celle de Lanoire, la première sur le plan chronologique, est celle qui s'écarte le plus de la perspective originelle alors que celle de Pellan est celle qui s'en rapproche le plus.

Quant à l'utilisation du concordancier, cet outil permet de retrouver plus rapidement et plus facilement les indices d'un passage en discours indirect libre; mais il appartient toujours au chercheur d'en interpréter l'effet sur l'univers fictif du récit. Des logiciels tels que Multiconcord et WordSmith Tools se bornent à relever des indices en fonction des critères définis par le chercheur; ils sont donc utiles pour un premier tri des données puisqu'ils relèvent non seulement les indices, mais aussi les contextes dans lesquels ceux-ci apparaissent. De plus, ils permettent, en une fraction de seconde, de comparer la traduction et le texte original. Là toutefois s'arrête leur utilité.

Le chapitre 6 enfin est consacré à l'analyse de The Waves (1931) ainsi qu'à sa traduction par Marguerite Yourcenar sous le titre de Les Vagues (1937) et à celle de Cécile Wasjbrot sous le même titre (1993). Le roman est écrit à la première personne par les six personnages qui discutent entre eux et un septième personnage qui demeure muet. Il comprend de nombreux monologues intérieurs dans lesquels les personnages se parlent à eux-mêmes ou parlent aux autres comme par télépathie, le tout interrompu de temps à autre par le narrateur. Il est intéressant de noter que si le texte de Woolf comprenait 78104 mots, la traduction de Yourcenar en comprend 91623 et celle de Wajsbrot 74 406. Si l'on tient compte du fait que, par convention, le français a tendance à éviter les répétitions qui, en anglais, servent à mettre en relief un aspect spatio-temporel du récit, on se rend compte que les deux traductrices ont dû faire appel à un vocabulaire plus varié que celui de Woolf pour éviter la répétition de divers pronoms ou tournures grammaticales, comme I, I am et I am not et que, les deux textes demeurant sensiblement près de l'original, le vocabulaire de Wasjbrot est sensiblement plus varié que celui de Yourcenar.

Comme elle l'avait fait au chapitre précédent, l'auteure procède en deux étapes, la première étant une analyse quantitative des indices déictiques, de modalité et de transitivité présents dans les textes, la deuxième, une analyse de huit paragraphes contenant un grand nombre d'indices.

On aurait pu croire que le soin mis à éviter les répétitions ou la non-traduction de certains indices déictiques pourrait avoir comme conséquence de modifier l'univers fictif des deux traductions. Il n'en est rien: Yourcenar rend plus systématiquement les déictiques 
et utilise davantage les mêmes verbes d'obligation et de permission que Wasjbrot, de telle sorte que même si son texte est sensiblement plus long et "plus français", l'univers fictif des personnages demeure semblable à celui de Woolf. La différence la plus marquée se situe sur le plan de la transitivité des verbes, Yourcenar utilisant au moins à une occasion la voix passive au lieu de la voix active ou passant d'une construction personnelle à une construction impersonnelle. Wajsbrot, pour sa part, se contente à quelques occasions de faire allusion au sujet de la phrase sans l'énoncer clairement. Toutefois, en raison du faible nombre d'occurrences et de l'absence de glissements véritables dans le texte, il n'en résulte guère de changements de perspective autres que des modifications dans la façon dont les personnages se perçoivent et perçoivent leurs relations avec l'univers qui les entoure.

Cette analyse des choix linguistiques des traductrices montre que toutes deux ont laissé leur empreinte distinctive sur le texte, mais de façon fort différente. Dans les deux cas toutefois, elles ont créé deux voix différentes qui se superposent tant aux voix des personnages qu'à la voix de l'auteure implicite du texte.

Comme dans le cas du premier roman, l'utilisation d'un corpus s'est avérée extrêmement utile en permettant d'identifier rapidement des éléments linguistiques impliquant une idée de perspective ainsi que leur contexte. Elle a permis d'identifier et d'analyser en profondeur quelque 443 exemples comprenant des éléments déictiques, de modalité et de transitivité beaucoup plus rapidement que ce n'eût été le cas de façon manuelle. Mais ici encore, l'utilisation de logiciels tels Multiconcord a dû se faire avec un œil critique, le logiciel traitant comme de nouvelles occurrences les homonymes et les répétitions. De plus, le logiciel ne s'avère pas d'un grand secours lorsque vient le temps d'analyser la signification de ces répétitions, pas plus que pour déterminer si un glissement sur le plan microstructurel affecte la perspective sur le plan macrostructurel.

En conclusion, l'auteure revient sur le problème posé par le fait que la narratologie n'établit que rarement une distinction entre original et traduction, occultant ainsi les traces laissées par le traducteur. Le modèle que présente Charlotte Bosseaux répond à cette question en découvrant la présence du traducteur grâce aux choix linguistiques affectant les structures narratives. Comme on pouvait s'y attendre, l'utilisation de techniques gérées par ordinateur a grandement facilité le travail en permettant une analyse exhaustive et en profondeur des textes en question. Mais si corpus et concordanciers permettent de retracer aisément divers indices comme les déictiques ou les éléments de modalité et de transitivité, ils s'avèrent déjà moins utiles pour déterminer quels passages sont en discours indirect libre puisque les mêmes indices peuvent se retrouver dans d'autres formes de discours. Enfin, seul le chercheur est en mesure de déterminer si les choix linguistiques et stylistiques du traducteur ont eu une influence sur l'univers fictif du récit.

Les résultats de l'étude pourront être jugés jusqu'à un certain point décevants puisque la seule conclusion manifeste est que les premières traductions s'éloignent davantage des originaux que les traductions plus récentes, un phénomène que l'auteure attribue surtout à une meilleure connaissance de Virginia Woolf en France et au fait que les derniers traducteurs étaient des spécialistes de Woolf. S’ajoute à cela le fait que Yourcenar était une auteure de plein droit et que la traduction ne pouvait se faire chez elle sans y allier création littéraire, alors que, de son propre aveu, le souci premier de Wasjbot était de se démarquer de la traduction précédente.

L'intérêt de ce livre réside donc moins dans la réponse apportée à la question formulée dans le titre que dans le processus d'analyse élaboré par Charlotte Bosseaux pour y arriver. En définissant très clairement ses concepts, en présentant en détail ses outils de travail et les étapes à franchir, en résumant chacune d'elles au fur et à mesure de la progression des chapitres, en résumant les conclusions pouvant être tirées à chaque stade de l'opération, Charlotte Bosseaux définit un modèle pouvant être reproduit et testé par ordinateur dans diverses circonstances, soit pour d'autres genres littéraires comme les articles de journaux, 
ou pour d'autres combinaisons linguistiques que l'anglais et le français, ouvrant ainsi de nouvelles perspectives de recherche et constituant un outil utile pour les traducteurs soucieux de mieux comprendre les techniques narratives et d'y adapter leurs stratégies de traduction.

Serge Marcoux

Université de Montréal, Montréal, Canada

\section{NOTE}

1. Gérard de Cortanze dans le Magazine Littéraire (1993) en dira: «Elle ôte les aspérités. Elle police plus qu'elle ne polit le texte, fait de ce parc anglais un jardin à la française» (traduction de Charlotte Brosseaux).

\section{RÉFÉRENCES}

BAKER, M. (2000): “Towards a methodology for investigating the style of a literary translator", Target 12-2, pp. 241-266.

Cortanze, G. de. (1993): «Virginia Woolf, instants de vie», Magazine Littéraire 309, pp. 9596.

Forrester, V. (1993): «Qui a trahi Virginia Woolf?», Le Monde, 23 avril 1993, p. 30.

Munday, J. (1998): "A computer-assisted approach to the analysis of translation shifts", Meta 43-3, pp. 1-16.

Munday, J. (2002): "Systems in translation: a systemic model for descriptive translation studies", in Hermans, T. (ed.), Crosscultural Transgressions. Research Models in Translation Studies II: Historical and Ideological Issues, Manchester, St Jerome, pp. 76-92.

Reynier, C. (ed.) (1993): Études Britanniques Contemporaines $n^{\circ} 2$, Montpellier, Presses universitaires de Montpellier.

Roy, P. (1977): The Dual Voice: Free Indirect Speech an its Functioning in the Nineteenth-Century European Novel, Manchester, Manchester University Press and Rowan and Littlefield.

Woolf, V. (1927): To the Lighthouse, London, Penguin Popular Classics.

Woolf, V. (1939): Moments of Being, in Schulkind, J. (ed.), London, Chatto and Windus, Sussex University Press.

JAnzen, T. (ed.) (2005): Topics in Signed Language Interpreting: Theory and Practice, Philadelphia, John Benjamins, 362 p.

Russell, Malcolm, Stratiy, Demers, les grands noms de la scène canadienne en matière d'interprétation en langue des signes, conjuguent leurs visions à celles de Wilcox et Shaffer du Nouveau-Mexique et de Leeson de Dublin dans un ouvrage qui est d'ores et déjà une référence pour qui veut cerner les problématiques essentielles du domaine. Terry Janzen, directeur de Topics in Signed Language Interpreting, présente l'état le plus achevé des réflexions théoriques et pratiques dans le champ de l'interprétation impliquant au moins une langue des signes au Canada. Au Canada anglais, devrions-nous préciser. Le livre se divise en trois parties. La première se résume à une introduction et une présentation des auteurs. La deuxième aborde le champ de la théorie et la troisième, celui de la pratique.

Les deux premiers textes de la section théorique traitent du processus cognitif qui préside à l'interprétation. Wilcox et Shaffer reformulent une présentation archiconnue des différents modèles utilisés pour représenter le rôle de l'interprète en langue des signes: modèle mécaniste, biculturel, sociolinguistique... S'ensuit une critique du concept de neutralité en interprétation que nous pourrions dépasser par une approche constructiviste 